\title{
Performance Comparison of Hybrid Wavelet Transform Formed by Combination of Different Base Transforms with DCT on Image Compression
}

\author{
Dr. H.B.Kekre \\ Sr. Professor, MPSTME, Department of Computer Engineering, NMIMS University, Mumbai, India \\ hbkekre@yahoo.com \\ Dr.TanujaSarode \\ Associate Professor Department of Computer Engineering, TSEC, Mumbai University, India \\ tanuja_0123@yahoo.com \\ PrachiNatu \\ Assistant Professor and Ph. D. Research Scholar, MPSTME, Department of Computer Engineering, NMIMS University, \\ Mumbai, India \\ prachi.natu@yahoo.com
}

\begin{abstract}
In this paper image compression using hybrid wavelet transform is proposed. Hybrid wavelet transform matrix is formed using two component orthogonal transforms. One is base transform which contributes to global features of an image and another transform contributes to local features. Here base transform is varied to observe its effect on image quality at different compression ratios. Different transforms like Discrete Kekre Transform (DKT), Walsh, Real-DFT, Sine, Hartley and Slant transform are chosen as base transforms. They are combined with Discrete Cosine Transform (DCT) that contributes to local features of an image. Sizes of component orthogonal transforms are varied as $16-16,32-8$ and $64-4$ to generate hybrid wavelet transform of size 256x256. Results of different combinations are compared and it has been observed that, DKT as a base transform combined with DCT gives better results for size $16 \times 16$ of both component transforms.
\end{abstract}

Index Terms - Hybrid wavelet transform, Real-DFT, Kekre transform, DCT.

\section{INTRODUCTION}

In recent years wide range of study has been done on wavelet transforms and their use in different applications. Increased use of images has made it compulsory to study the use of wavelets in image compression application. Plain areas of images contain high degree of redundancy. Also human visual system is able to tolerate some distortion in the image. By considering these facts, it is possible to eliminate the redundancies in the image and compress it. It saves amount of storage space as well as time required for transmission. Transform based coding is popular technique to de-correlate the pixels. Transform converts the signal into frequency domain and makes processing of signal easier. Initially Discrete Fourier Transform was proposed. But it cannot detect local information in the signals. Dennis Gabor introduced a solution to overcome this drawback. It is known as Short Time Fourier Transform (STFT) orwindowedtransform [1]. Later on it was named as Gabor Transform. It gives local properties at the cost of global properties. Effective transforms that came into picture for image compression are Discrete Cosine Transform (DCT) [2] and wavelet transform. DCT shows good energy compaction property. Image is first divided into NxN size blocks and DCT is applied on each block individually. But it introduces blocking effect at the edges of the image. Larger the block size more is the blockingeffect.Inrecent two to three decades research has been done on wavelet transforms. It proves to be better than DCT in the sense that it is applied on the whole image and not on individual block. Hence there is no blocking effect in wavelet transform and compression error is distributed uniformly over entire image. It provides higher compression ratio and better image quality than DCT $[3,4]$. Hence it is more robust under transmission and decoding errors [5].Initial focus was on Haar wavelet. But now different wavelet transforms of Walsh, Kekre, Slant, Hartley, Sine and Real DFT [6,7] also have been studied. As each wavelet transform has its own features suitable for specific applications, there is a scope to study the use of transforms obtained by combining two different transforms. Latest trend is to use such hybrid wavelet transforms in image processing applications. 
Remaining paper is organized as follows: Section II presents work done in the field of image compression. Section III gives proposed algorithm. Experiments and results are discussed in section IV and conclusion is drawn in section $\mathrm{V}$.

\section{RELATED WORK}

A lot of work has been done on image compression and still it is going on. Haar wavelets have been popularly used for image compression. Analysis of different wavelets for image compression have been done in [8] byPrabhakaret al. and it has been observed that Haar wavelets perform better than other wavelets like Symlet, coiflet and db4 wavelets. In [9] Yi Zhang has proposed fractal image compression based on wavelet using diamond search. But the drawback over here is fractal image coding takes more time. Wavelet based image compression followed by EZW coding is proposed in [10]. Combination of these two gives better compression ratio. Other hybrid techniques which combine two different techniques are proposed in recent research to obtain better results.

A Neuro-Wavelet based approach for image compression is proposed by Singh et al. in [11]. Images are decomposed using wavelet filters into a set of sub bands with different resolution corresponding to different frequency bands. Different quantization and coding schemes are used for different sub bands based on their statistical properties. The coefficients in low frequency band are compressed by differential pulse code modulation (DPCM) and the coefficients in higher frequency bands are compressed using neural network. Using their proposed scheme one can accomplish satisfactory reconstructed images with large compression ratios. Combination of vector quantization and wavelet transform using RBF neural network has been proposed in [12]. Bi-orthogonal wavelet based image compression combined with hierarchical back propagation neural network is presented in [13] But complexity of such techniques is also more. Hybrid wavelet transform is much simpler technique which can be applied for image compression application as mentioned in [14]. It uses two different orthogonal component transforms to form hybrid wavelet transform matrix. It contains properties of both the component transforms and operates on entire image.

This paper extends the study of hybrid wavelet transform presented in [14] to observe the effect of selecting different component transforms to contribute global features. Hybrid wavelet transform matrix of image size is created and applied on each image. Two orthogonal component transforms are used to create hybrid transform. First component transform contributing to global features is called base transform which is varied and second component transform is selected as Discrete Cosine Transform (DCT). Three different sizes of component transform are considered. Base transform of size $16 \times 16,32 \times 32$ and $64 \times 64$ is combined with second component transform of size $16 \times 16,8 \times 8$ and $4 \times 4$ respectively to obtain hybrid wavelet transform of image size i.e. 256x256. Hybrid wavelet is generated using the procedure mentioned in [14].

\section{PROPOSED ALGORITHM}

1. Consider colour image of size $256 \times 256$.

2. Separate R, G, B components of an image

3. Base transform of size $16 \times 16$ and second component transform 'DCT' of size $16 \times 16$ is selected to obtain hybrid transform of $256 \times 256$ sizes. Base transform is varied as DKT, Walsh, Real-DFT, Sine, Hartley and Slant transform to compare their performances.

4. Full Hybrid Wavelet Transform of image ' $\mathrm{f}$ ' is obtained as ' $\mathrm{F}$ ' $=[\mathrm{T}] *[\mathrm{f}] *[\mathrm{~T}]^{\mathrm{T}}$.

5. Elements in transformed plane are sorted in descending order of their energy and in each step (8x256) lowest energy elements are eliminated.

6. Reconstruct the image by applying inverse transform.

7. Calculate RMSE and PSNR between original image and reconstructed image at different compression ratios.

8. Vary the size of base transforms mentioned in step 3, to 32x32 and 64x64 and that of second transform to $8 \times 8$ and $4 \times 4$. Regenerate hybrid wavelet transform matrix T. Repeat steps 4 to 7 using this transform matrix. Compare the results in each case.

\section{EXPERIMENTS AND RESULTS}

Proposed technique is applied on a set of twelve colour images of size 256x256. Experiments are performed using Matlab 7.0 on AMD dual core processor with 4 GB RAM. Images chosen for experimental work are shown in Fig. 1. 

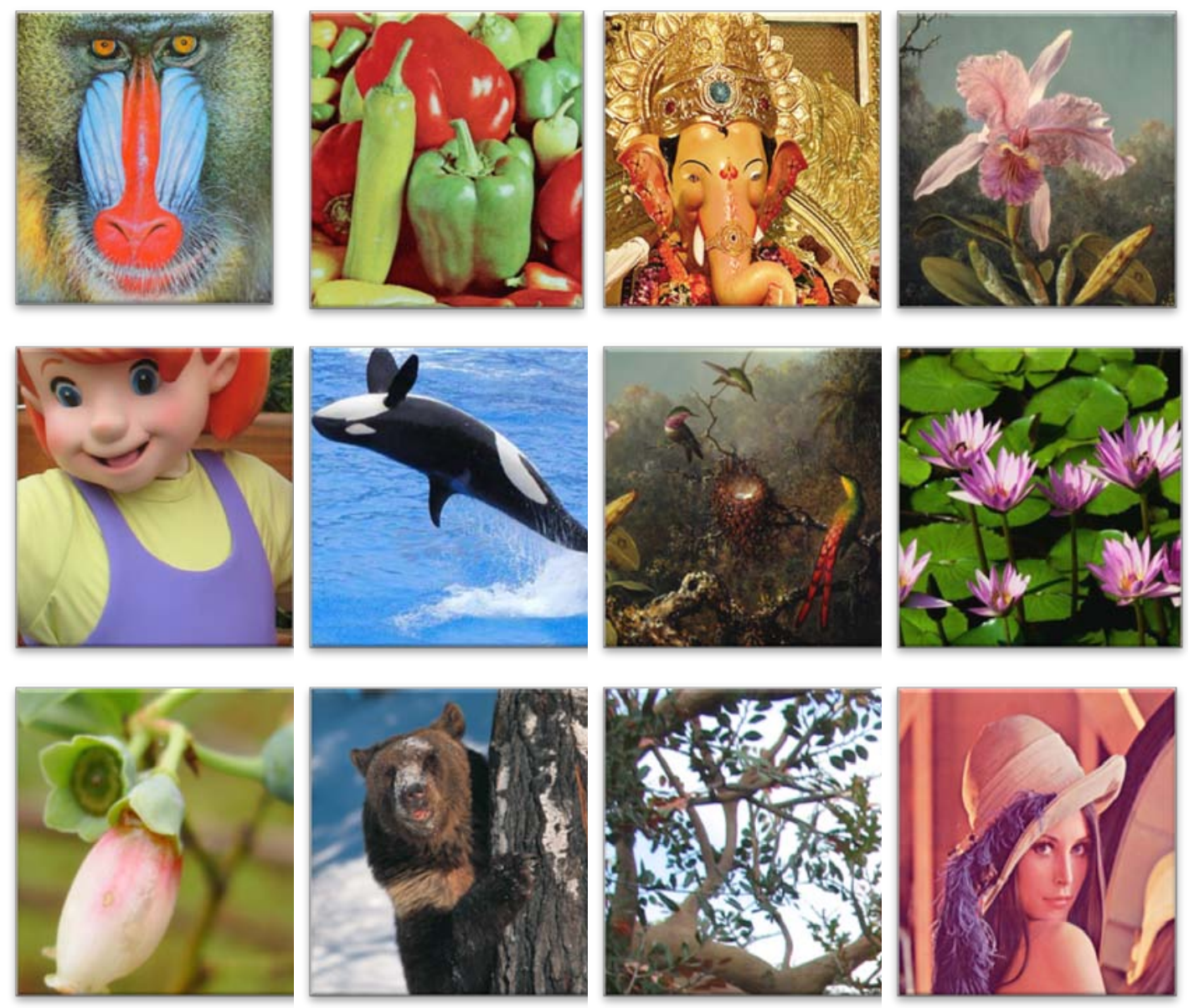

Figure 1. Set of twelve test images of different classes used for experimental purpose namely (from left to right and top to bottom) Mandrill, Peppers, Lord Ganesha, Flower, Cartoon, Dolphin, Birds, Waterlili, Bud, Bear, Leaves and Lena

Fig. 2 shows average RMSE versus Compression ratio for different hybrid wavelet transforms. Here base transform is varied and second component transform is selected as DCT. Size of base transform and DCT is selected as $16 x 16$. In each case results are observed.
From graph it can be observed that DKT-DCT hybrid wavelet gives better results than other combinations with DCT. At higher compression ratios more difference in RMSE can be seen.

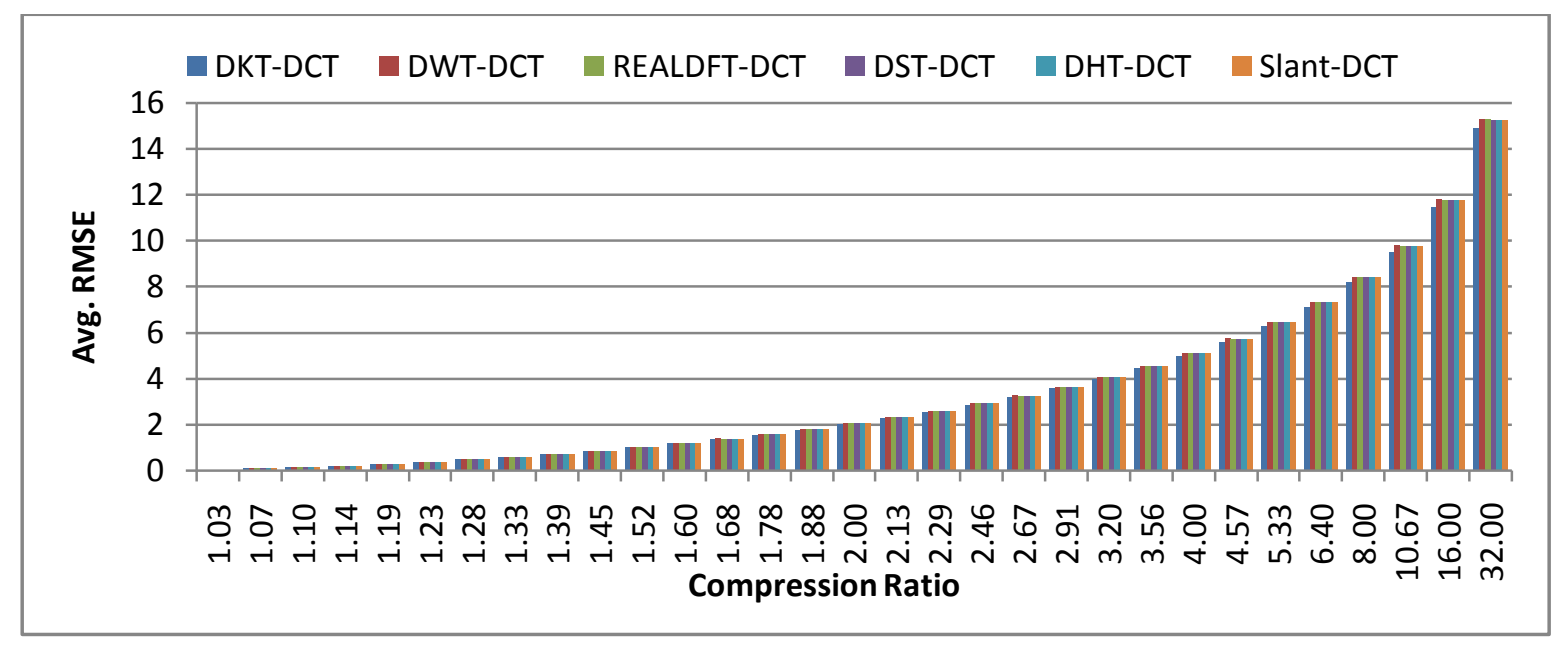

Figure 2. Average RMSE at different compression ratios by using different base transforms of size 16x16 with DCT of size 16x16 in hybrid wavelet transform. 
42 Performance Comparison of Hybrid Wavelet Transform Formed by Combination of Different Base Transforms with DCT on Image Compression

Fig.3 shows three sample reconstructed images namely Peppers, Mandrill and Flower respectively obtained by applying hybrid wavelet transform. Base transform is varied and its size is selected as $16 \times 16$. Size of second component transform i.e. DCT is also selected as $16 x 16$. From reconstructed images it can be seen that DKT-DCT hybrid wavelet gives better results as compared to other combinations.
In Fig.4, graph shows average RMSE versus compression ratio. Here base transform size is changed to $32 \mathrm{x} 32$ and DCT matrix of size $8 \mathrm{x} 8$ is selected. Here also DKT-DCT performs better than all other transforms. In addition difference between the performance of DKT-DCT and other transforms is more than the previous case.

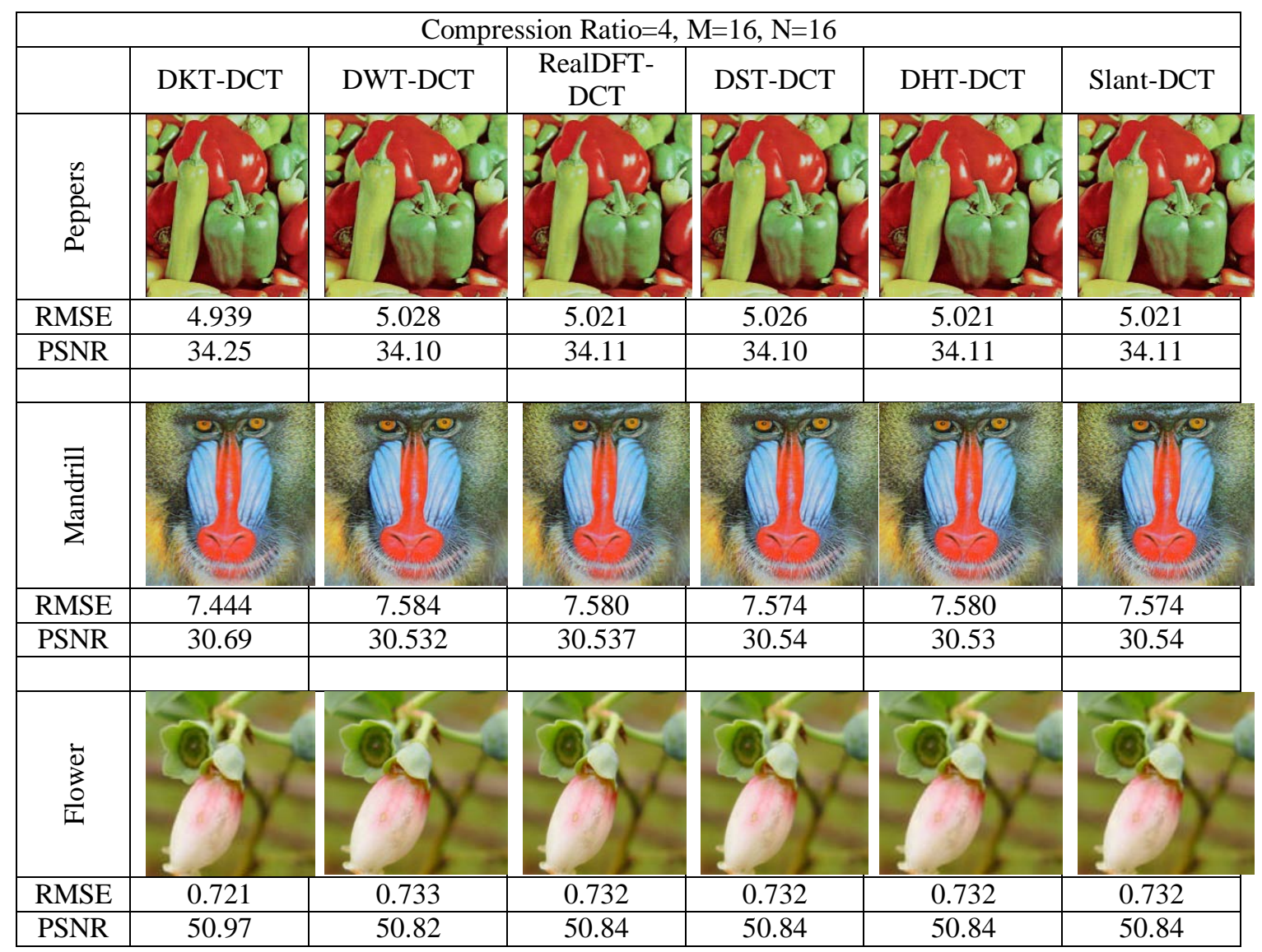

Figure 3. Reconstructed images using different hybrid wavelet transforms at compression ratio 4 with base component transform of size $16 \times 16$

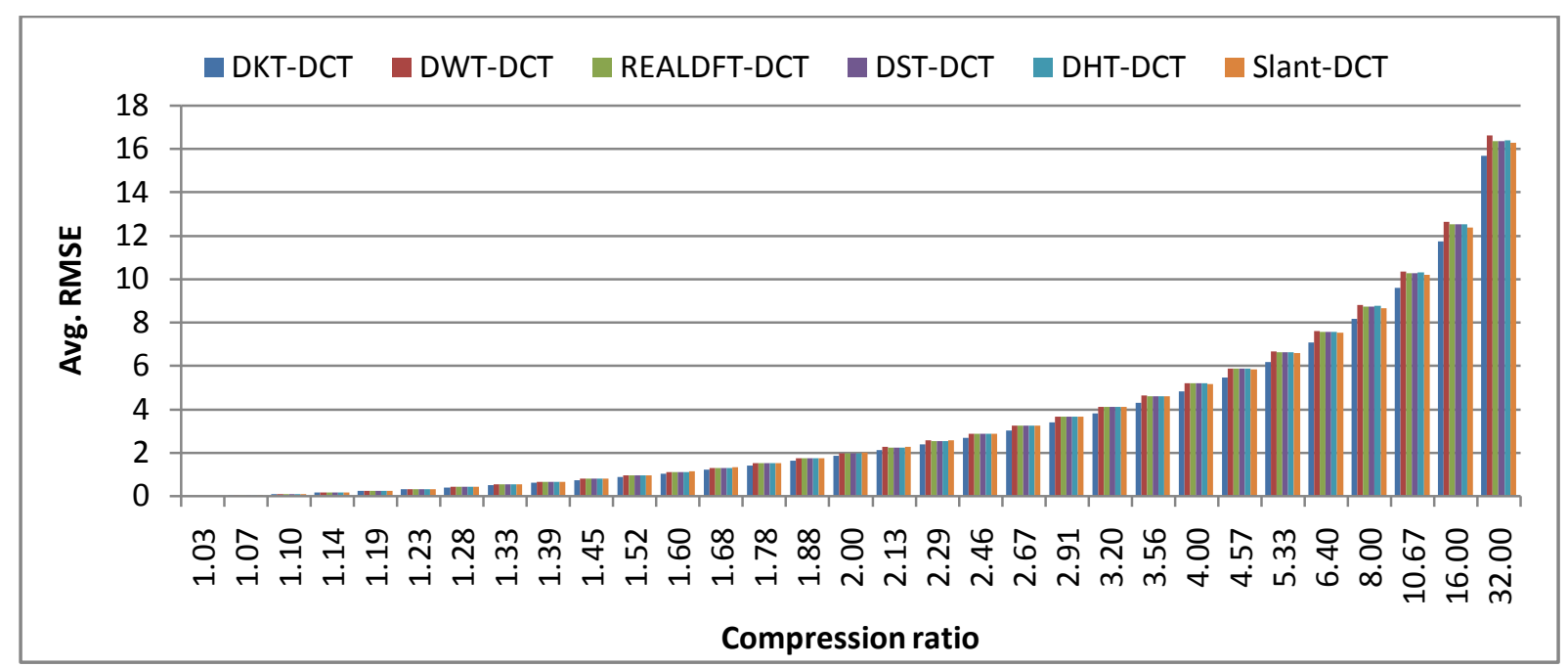

Figure 4. Average RMSE at different compression ratios by using different base transforms of size 32x32 with DCT of size 8x8in hybrid wavelet transform. 
Fig. 5 shows comparison of RMSE and PSNR values for three sample images namely Peppers, Mandrill and Flower. Base transform size is $32 \times 32$ and DCT is of size 8x8. DKT -DCT shows better results in this case also.

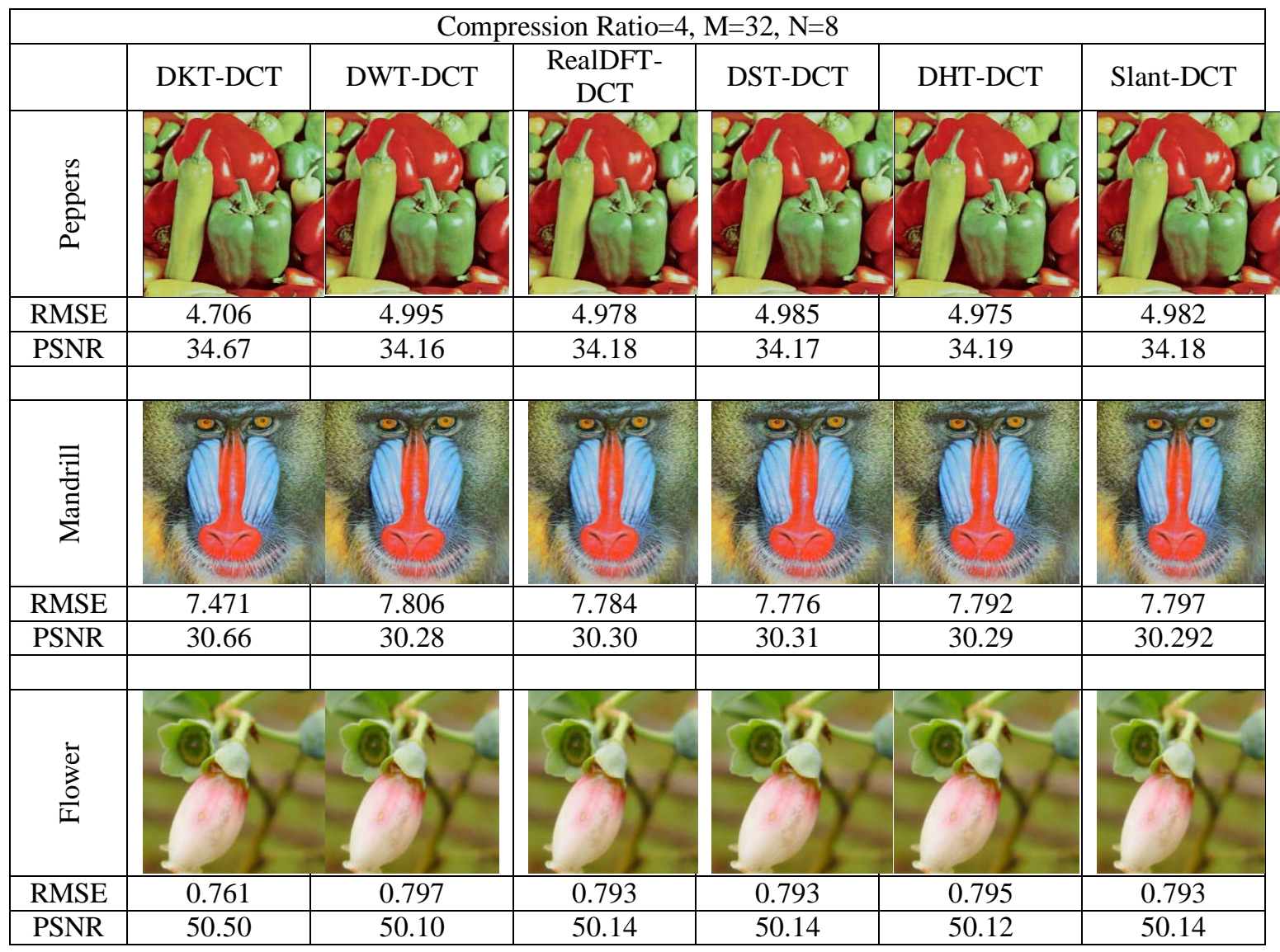

Figure 5. Reconstructed images using different hybrid wavelet transforms at compression ratio 4 with base component transform of size $32 \times 32$

In Fig. 6 RMSE is plotted for base transform size $64 \times 64$ and DCT matrix of 4x4. In this case also DKTDCT shows superior performance except at compression ratio 32. RealDFT-DCT gives minimum value of RMSE than others at compression ratio 32 . DKT-DCT shows sudden rise in RMSE values than in previous case.

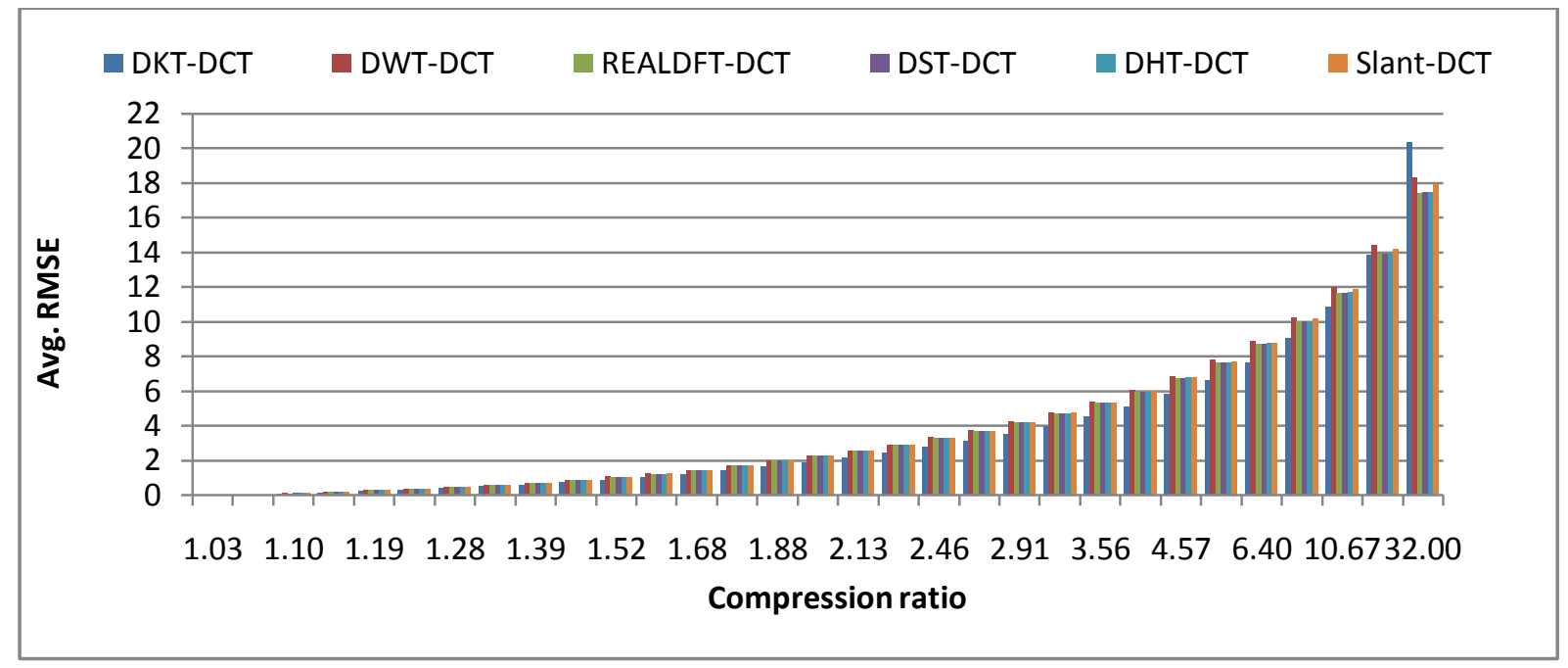

Figure 6. Average RMSE at different compression ratios by using different base transforms of size $64 x 64$ with DCT of size $4 \times 4$ in hybrid wavelet transform. 
44 Performance Comparison of Hybrid Wavelet Transform Formed by Combination of Different Base Transforms with DCT on Image Compression

Reconstructed sample images corresponding to graph in Fig 6 are shown in Fig.7. Noticeable difference is observed between DKT-DCT and other hybrid transforms.

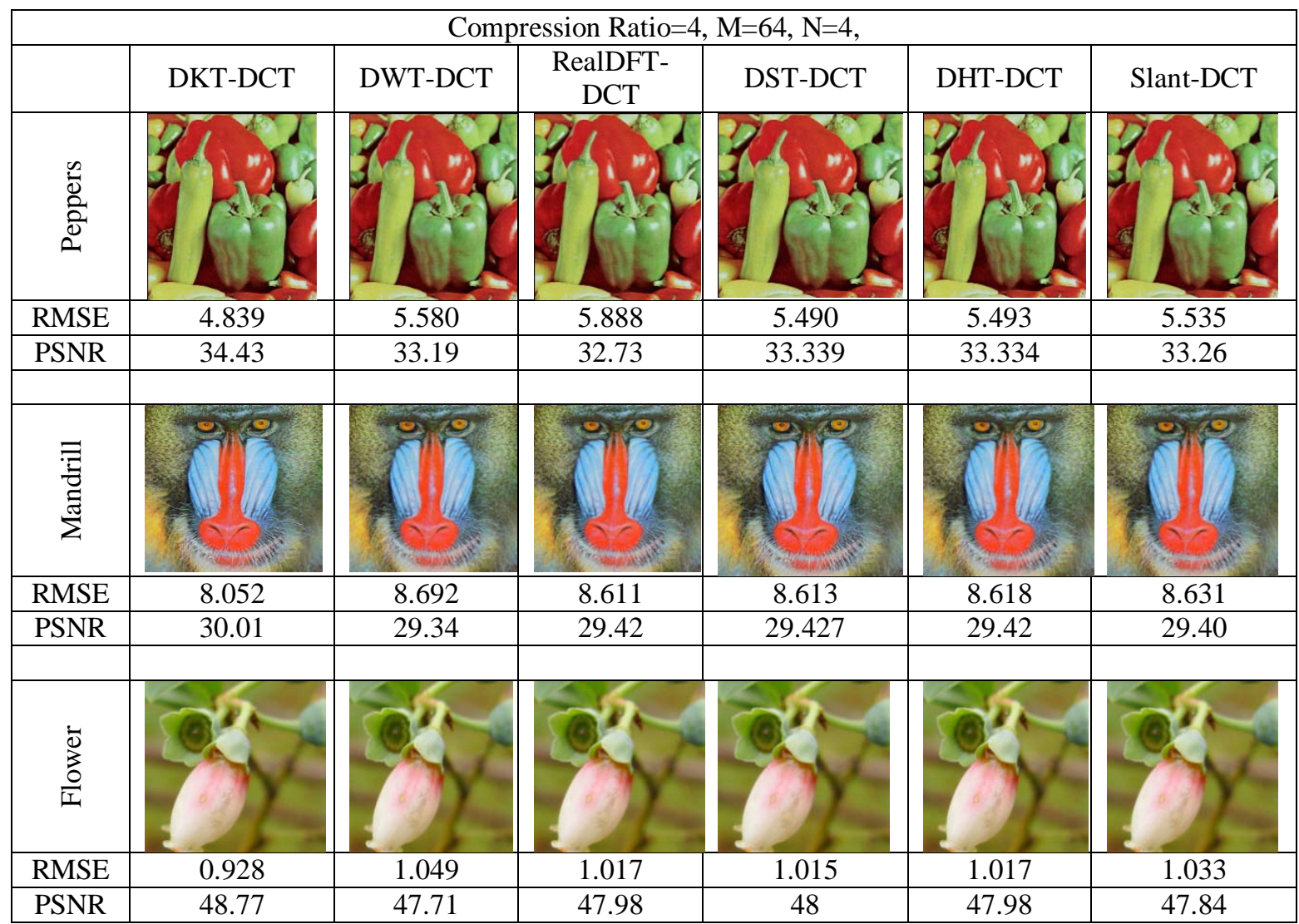

Figure 7. Reconstructed images using different hybrid wavelet transforms at compression ratio 4 with base component transform of size $64 \times 64$

\section{CONCLUSION}

In this paper, performance of different hybrid wavelet transforms has been compared. Two component orthogonal transforms of different sizes are used to form hybrid wavelet transform. First component transform called base transform is varied and second component transform is selected as DCT. Effect of variation in base transform with different size is observed in terms of root mean square error. It has been observed that DKT-DCT combination gives better performance even at higher compression ratios. Even with variation in size of base transform, DKT-DCT proves to be superior in performance. All in average DKT-DCT performs better compared to all other combinations at size $16 \times 16$ of both component transforms.

\section{REFERENCES}

[1] M. Sifuzzaman, M.R.Islam, M.Z. Ali, "Application of Wavelet transform and Its Advantages Compared to Fourier Transform”, Journal of Physical Sciences, Vol. 13, 2009, 121134.
[2] N. Ahmed, T. Natarajan and K. R. Rao, "Discrete Cosine Transform”, IEEE Transaction Computers, C-23, pp. 90-93, January 1974.

[3] Sonja Grgic, MislavGrgic,, and BrankaZovkoCihlar, "Performance Analysis of Image Compression using Wavelets”, IEEE Transactions On Industrial Electronics, Vol. 48, No. 3, June 2001.

[4] M. J. Nadenau, J. Reichel, and M. Kunt, "Wavelet Based Color Image Compression: Exploiting the Contrast SensitivityFunction,” IEEE Transactions Image Processing, Vol.12, no.1, pp. 58-70, 2003.

[5] H.B.Kekre,TanujaSarode, PrachiNatu, "Image Compression Based on Hybrid Wavelet Transform Generated using Orthogonal Component Transforms of Different Sizes.”, International Journal of Soft Computing and Engineering, (IJSCE), Vol. x, Issue X, July 2013, pp.

[6] H. B. Kekre, TanujaSarode, PrachiNatu, “ Image Compression Using Column, Row and Full Wavelet Transforms Of Walsh, Cosine, Haar, Kekre, Slant and Sine and Their Comparison With Corresponding Orthogonal Transforms", International Journal of Engineering Research and 
development (IJERD), Vol. 6, Issue 4, March 2013, pp.102-113.

[7] H.B.Kekre, TanujaSarode, PrachiNatu, "Image Compression using Real Fourier Transform, It's Wavelet Transform and Hybrid Wavelet with DCT”, International Journal of Advanced Computer Science and Applications,(IJACSA) Vol. 4, No.5, 2013, pp. 41-47.

[8] PrabhakarTelagarapuet al. "Image Compression using DCT and Wavelet Transformations", International Journal of Signal Processing, Image Processing and Pattern Recognition, Vol. 4, No. 3, September 2011, pp. 61- 74.

[9] Yi Zhang and Xing Yuan Wang "Fractal Compression Coding Based on Wavelet Transform with Diamond Search" Nonlinear analysis: Real World Applications, Vol. 13 Issue 1, Feb. 2012, pp. 106-112.

[10] V.V. Sunil Kumar, M. IndraSena Reddy, "Image Compression Technique by using Wavelet Transform”, Journal of Information Engineering and Applications, Vol.2, No.5, 2012, pp.35-39.

[11] V.Singh, N. Rajpal, and K. S. Murthy, "NeuroWavelet Based Approach for Image Compression”, Computer Graphics, Imaging and Visualization, CGIV apos'2007, pp. 280-286.

[12] G. Boopathi, Dr. S. Arockiasamy, "Image Compression: Wavelet Transform using Radial Basis function (RBF) Neural Network", IEEE Transactions, 2012, pp. 340-344.

[13] Anchal Rani, Mohit Mehta, “ Image Compression of Radiograph using Neural Network and Wavelet”, International Journal of Engineering Science and Technology (IJEST), Vol. 5, No. 4, April 2013, pp. 803-809.

[14] H.B. Kekre, TanujaSarode, SudeepThepade, "Inception of Hybrid Wavelet Transform using Two Orthogonal Transforms and It's Use For Image Compression”, International Journal of Computer Science and Information Security(IJCSIS),Vol. 9, No. 6, 2011, pp. 80-87.

\section{AUTHORS PROFILE}

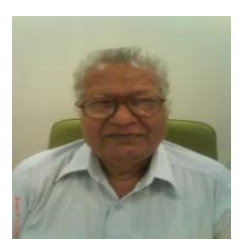

Dr. H. B. Kekre has received B.E (Hons.) in Telecomm Engineering from Jabalpur University in 1958, M.Tech (Industrial Electronics) from IIT Bombay in 1960, He has guided 17 Ph.Ds, more than 100 M.E./M.Tech and several B.E. / B.Tech projects, while in IIT and TSEC. His areas of interest are Digital Signal processing, Image Processing and Computer Networking. He has more than 450 papers in National / International Journals and Conferences to his credit. He was Senior Member of IEEE. Presently He is Fellow of IETE, Life Member of ISTE and Senior Member of International Association of Computer Science and
Information Technology (IACSIT). Recently fifteen students working under his guidance have received best paper awards. Currently eight research scholars working under his guidance have been awarded $\mathrm{Ph}$. $\mathrm{D}$. by NMIMS (Deemed to be University). At present eight research scholars are pursuing Ph.D. program under his guidance.

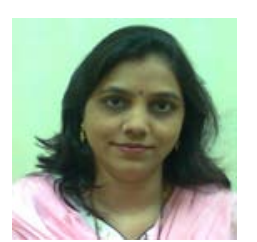

Dr.Tanuja K. Sarodehas received M.E. (Computer Engineering) degree from Mumbai University in 2004, Ph.D. from Mukesh Patel School of Technology, Management and Engg.SVKM's NMIMS University, Vile-Parle (W), Mumbai, INDIA. She has more than 11 years of experience in teaching. Currently working as Assistant Professor in Dept. of Computer Engineering at ThadomalShahani Engineering College, Mumbai. She is member of International Association of Engineers (IAENG) and International Association of Computer Science and Information Technology (IACSIT). Her areas of interest are Image Processing, Signal Processing and Computer Graphics. She has 150 papers in National /International Conferences/journal to her credit.

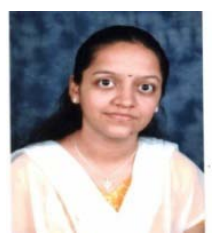

Ms.Prachi Natu has received M.E. (Computer) degree from Mumbai University in 2011. Currently pursuing Ph.D. from NMIMS University. She has 08 years of experience in teaching. Currently working as Assistant Professor in Department of Computer Engineering at Mukesh Patel School of Technology, Management and Engineering.Vile Parle (Mumbai). Her areas of interest are Image Processing, Database Management Systems and Operating Systems. She has 18 papers in International Conferences/journal to her credit. 